\title{
The lipid-lowering effects of Danhong and Huangqi injections: a meta-analysis of clinical controlled trials
}

\author{
Liuqin $\mathrm{Yu}^{1 \dagger}$, Chunyang Zhou ${ }^{1 \dagger}$, Zhi Luo ${ }^{2}$, Wei Zeng ${ }^{2}$, Feiya Lai ${ }^{3}$, Gangjie Han ${ }^{3}$ and Yongyan Song ${ }^{4^{*}}$
}

\begin{abstract}
Background: Dyslipidaemia is a major risk factor for coronary heart disease (CHD). Danhong and Huangqi injections, two traditional Chinese medicine prescriptions, have been widely studied regarding their lipid-lowering properties. However, the results were inconsistent and inconclusive. Thus, we conducted this meta-analysis of clinical controlled trials to clarify the lipid-lowering effects of Danhong and Huangqi injections.

Methods: The databases including PubMed, Google Scholar, Web of Science, Cochrane Library, Wanfang Database, CNKI and VIP were searched. The following information was obtained from each study: first author, age, gender, ethnicity, health condition, treatment dose, treatment duration, sample size, mean and standard deviation or standard error of lipid variables before and after treatment. The changes in lipid levels from pre- to post-treatment were calculated and compared between the control groups and the treatment groups in this meta-analysis.

Results: Forty-four studies (5021 subjects) and 7 studies (542 subjects) were respectively identified for Danhong and Huangqi injections. Compared with the control groups, Danhong injection yielded a significant reduction in triglycerides (TG) [standardized mean difference $(\mathrm{SMD})=-0.76,95 \%$ confidence interval $(\mathrm{Cl})=(-0.91,-0.61), P<0$. $001]$, total cholesterol (TC) [SMD $=-1.29,95 \% \mathrm{Cl}=(-1.56,-1.03), P<0.001]$ and low-density lipoprotein cholesterol (LDL-C) [SMD $=-0.76,95 \% \mathrm{Cl}=(-0.93,-0.59), P<0.001]$, and a significant elevation in high-density lipoprotein cholesterol (HDL-C) $[S M D=0.70,95 \% \mathrm{Cl}=(0.41,0.98), P<0.001]$. Regarding Huangqi injection, it yielded a significant reduction in $\mathrm{TC}[\mathrm{SMD}=-1.13,95 \% \mathrm{Cl}=(-2.09,-0.16), P=0.02]$ and marginally in $\mathrm{TG}[\mathrm{SMD}=-1.27$, $95 \% \mathrm{Cl}=(-2.53,0.00), P=0.05]$ comparing with the control groups.

Conclusions: Danhong injection can effectively decrease the plasma levels of TG, TC and LDL-C, and increase HDL$\mathrm{C}$ levels. Huangqi injection also has significant effects on TG and TC reduction, but not as powerful as Danhong injection.
\end{abstract}

Keywords: Danhong injection, Huangqi injection, Lipid, Dyslipidaemia, CHD

\section{Background}

Dyslipidaemia is a common lipid disorder characterized by increased levels of triglycerides (TG), total cholesterol (TC) and low-density lipoprotein cholesterol (LDL-C), and/or decreased level of high-density lipoprotein cholesterol (HDL-C) in circulation. Dyslipidaemia is one of the most important risk factors for coronary heart

\footnotetext{
* Correspondence: songyongyan2014@foxmail.com

†Equal contributors

${ }^{4}$ School of Preclinical Medicine, North Sichuan Medical College, and

Nanchong Industry Technology Institute of BioMedicine, Nanchong 637000 ,

People's Republic of China

Full list of author information is available at the end of the article
}

disease (CHD) and accounts for at least $50 \%$ of the population-attributable risk [1]. A review of randomized controlled trials (RCTs) concluded that a $40 \%$ reduction in LDL-C levels and a 30\% increase in HDL-C levels could lower the CHD risk by $70 \%$ [2]. To date, several classes of lipid-lowering drugs have been introduced: inhibitors of 3-hydroxy-3-methylglutaryl coenzyme A reductase (e.g. statins), inhibitors of intestinal cholesterol absorption (e.g. ezetimibe), bile acid sequestrants (e.g. chlestyramine), anti-PCSK9 monoclonal antibodies (e.g. evolocumab), and so on, which have considerable benefits for improving serum lipid profiles but also have a 
number of drawbacks. For example, statins were reported to give rise to some serious adverse effects such as inflammatory and necrotizing myopathies [3-5]. Due to these concerns, there have been increasing attempts to use functional natural products as alternatives to the conventional lipid-modulating agents, which are often more acceptable to patients.

Danhong and Huangqi injections are two traditional Chinese medicines being widely used in clinical practice in China. Danhong injection is a herbal product from radix salviae miltiorrhizae and flos carthami. The main components of Danhong injection include tanshinone, salvia acid, salvianolic acid, safflower yellow pigment, safflower phenolic glycosides, catechol and so on. Clinical studies have shown that Danhong injection was effective in the treatment of CHD [6, 7], cerebral infarction [8] and hepatic veno-occlusive disease [9]. Huangqi injection (also known as astragalus injection) is made from radix astragali. Its main components are astragalosides, polysaccharides, flavones, amino acids and so on. Several researches concluded that Huangqi injection was effective in the treatment of aplastic anemia [10], diabetic nephropathy [11] and leucopenia [12]. The use of radix salviae miltiorrhizae, flos carthami and radix astragali was firstly described in the Chinese ancient medical books such as materia medica, Shen Nong Ben Cao Jing and Kai Bao Ben Cao. Currently, they are officially listed in the Chinese Pharmacopoeia.

A large number of studies have investigated the effects of Danhong and Huangqi injections on plasma lipid levels, but the results were inconsistent and inconclusive. In some of these studies, Danhong injection was reported to decrease plasma levels of TG [13-39], TC [16-43] and LDL-C [15, 17-33, 36-41, 44, 45], and increase HDL-C levels [17-32, 35, 40]; Huangqi injection was reported to decrease plasma levels of TG [46-51], TC [47-52] and LDL-C [48, 51], and increase HDL-C levels [51]. However, the results from other studies did not support these findings [53-56]. Therefore, a metaanalysis is required to clarify the lipid-lowering capacity of the two injections so that they can be better used in clinical practice. In the current study, we conducted a systematic review of randomized clinical trials to explore the effects of Danhong and Huangqi injections on plasma lipid levels. Our analysis results can provide a reference for future clinical practice.

\section{Results}

\section{Characteristics of the included studies}

The initial search of the databases yielded 5236 articles. Four thousand nine hundred and seventy-eight articles were excluded according to titles and abstracts. Then full-text articles were retrieved and assessed on the basis of the inclusion criteria. Two hundred and seven articles were ineligible for the following reasons: 187 articles did not provide lipid data; 12 articles provided incomplete lipid data; 8 articles just compared the lipid-lowering effects of Danhong or Huangqi injection with other drugs. In the end, 51 studies [13-63] were selected for this meta-analysis, and all of which were published in Chinese.

The characteristics of the 51 studies are summarized in Additional file 1: Table S1. Forty-four studies [13-45, 53-63] reported lipid data for Danhong injection, and 7 studies [46-52] reported lipid data for Huangqi injection. For Danhong injection, 38 studies [13-39, 42, 43, 54-62], 41 studies [13, 14, 16-43, 53-63], 38 studies $[13-15,17-34,36-42,44,45,53,56-62]$ and 32 studies $[13-15,17-35,37-40,56-60,62]$ presented the data for TG, TC, LDL-C and HDL-C, respectively. For Huangqi injection, 6 studies [46-51], 7 studies [46-52], 3 studies $[46,48,51]$ and 3 studies $[46,48,51]$ presented the data for TG, TC, LDL-C and HDL-C, respectively. Nine studies $[15,19,20,24,27,45,54,56,60], 21$ studies $[17,18$, $21,22,25,26,28,31,32,34,35,37,38,40,41,48,57$, 58, 61-63], 5 studies [13, 23, 33, 42, 59], 9 studies [14, $16,29,30,36,39,43,44,55], 5$ studies [47, 49-52] and 2 studies $[46,53]$ involved CHD, stroke, hyperlipidemia, diabetes, nephrotic syndrome and hypertension, respectively. Thirty studies $[13,16-25,27-30,34-36,40,42-$ $45,53-56,58,60,63]$ and 14 studies $[14,15,26,31-33$, $37-39,41,57,59,61,62]$ respectively used high-dose treatment $(>20 \mathrm{~mL})$ and low-dose treatment $(\leq 20 \mathrm{~mL})$ with Danhong injection. Four studies [46, 47, 49, 51] and 3 studies $[48,50,52]$ respectively used high-dose treatment $(\geq 40 \mathrm{~mL})$ and low-dose treatment $(<40 \mathrm{~mL})$ with Huangqi injection. Twenty studies [13, 15, 19-21, $23,27,30,37-41,43,53,56-58,61,62]$ and 23 studies $[14,16-18,22,24-26,28,29,31-33,35,36,42,44,45$, $54,55,59,60,63]$ respectively used long-duration treatment ( $>2$ weeks) and short-duration treatment ( $\leq$ 2 weeks) with Danhong injection. Five studies [46-48, 51, 52] and 2 studies [49,50] respectively used longduration treatment ( $\geq 4$ weeks) and short-duration treatment $(<4$ weeks) with Huangqi injection. All the subjects included in the present meta-analysis were Chinese.

\section{Summary statistics}

Five thousand and twenty-one subjects and 542 subjects were respectively enrolled in the analyses for Danhong and Huangqi injections. For Danhong injection, $49.7 \%$ of the subjects (2495 subjects) were controls, and $50.3 \%$ of them (2526 subjects) were treated with Danhong injection. For Huangqi injection, $49.3 \%$ of the subjects $(267$ subjects) were controls, and $50.7 \%$ of them (275 subjects) were treated with Huangqi injection. Four thousand two hundred and thirteen, 4781, 4412 and 
3616 subjects were respectively included to compare the changes in TG, TC, LDL-C and HDL-C for Danhong injection (Aditional file 1: Table S1). Four hundred and forty, 542, 220 and 220 subjects were respectively included to compare the changes in TG, TC, LDL-C and HDL-C for Huangqi injection (Aditional file 1: Table S2).

\section{Associations of Danhong injection with plasma lipid levels}

The outcomes of the analysis on all studies showed that Danhong injection could effectively decrease the plasma levels of $\mathrm{TG}[\mathrm{SMD}=-0.77$, 95\% CI $=(-0.98,-0.56), P<0.001]$, TC $[$ SMD $=$ $-1.04,95 \% \mathrm{CI}=(-1.30,-0.78), P<0.001]$ and LDL-C $[\mathrm{SMD}=-0.69,95 \% \mathrm{CI}=(-0.87,-0.52), P<0.001]$, and increase HDL-C levels [SMD $=0.47,95 \% \mathrm{CI}=(0.19,0.75)$, $P<0.001$ ] (Table 1 and Figs. 1, 2, 3 and 4).

In the subgroup analyses stratified by health status, Danhong injection had significant effects on all of the four lipid parameters in CHD patients [TG: $\mathrm{SMD}=-1.04, \quad 95 \% \mathrm{CI}=(-1.30,-0.78), \quad P<0.001$; TC: $\mathrm{SMD}=-1.04,95 \% \mathrm{CI}=(-1.30,-0.78), P<0.001$; LDL-C: $\mathrm{SMD}=-1.04,95 \% \mathrm{CI}=(-1.30,-0.78), P<0.001$ and HDL-C: $\mathrm{SMD}=-1.04,95 \% \mathrm{CI}=(-1.30,-0.78), P$ $<0.001$ ] and in stroke patients [TG: SMD $=-0.94,95 \%$ $\mathrm{CI}=(-1.13,-0.74), P<0.001 ; \mathrm{TC}: \mathrm{SMD}=-1.53,95 \%$ $\mathrm{CI}=(-1.94,-1.13), \quad P<0.001 ;$ LDL-C: SMD $=-0.94$, 95\% CI $=(-1.24,-0.64), P<0.001$; HDL-C: SMD = 0.98, $95 \% \mathrm{CI}=(0.55,1.41), P<0.001]$. Danhong injection could significantly decrease TG, TC and LDL-C levels, but not HDL-C levels in diabetic patients [TG: SMD $=-0.58,95 \%$ $\mathrm{CI}=(-0.87,-0.29), P<0.001 ; \mathrm{TC}: \mathrm{SMD}=-1.28,95 \%$ $\mathrm{CI}=(-1.87,-0.70), \quad P<0.001 ;$ LDL-C: SMD $=-0.56$, 95\% CI $=(-0.86,-0.26), P<0.001]$ and in hyperlipidemic patients [TG: $\mathrm{SMD}=-0.87,95 \% \mathrm{CI}=(-1.54,-0.20)$, $P<0.001$; TC: SMD $=-1.16,95 \% \mathrm{CI}=(-1.46,-0.86)$, $P<0.001 ; \mathrm{LDL}-\mathrm{C}: \mathrm{SMD}=-0.54,95 \% \mathrm{CI}=(-0.74,-0.34)$, $P<0.001]$.

In the subgroup analyses stratified by the dose of the treatment with Danhong injection, both low- and highdose of treatment could significantly decrease the levels of TG [low-dose: SMD $=-0.90,95 \% \mathrm{CI}=(-1.15,-0.66)$ , $P<0.001$; high-dose: SMD $=-0.69,95 \% \mathrm{CI}=(-0.88$, $-0.50), P<0.001$ ], TC [low-dose: SMD $=-1.13,95 \% \mathrm{CI}$ $=(-1.49,-0.78), P<0.001 ;$ high-dose: $\mathrm{SMD}=-1.38$, 95\% CI $=(-1.73,-1.02), P<0.001]$ and LDL-C [lowdose: $\mathrm{SMD}=-0.79,95 \% \mathrm{CI}=(-1.05,-0.53), P<0.001$; high-dose: $\mathrm{SMD}=-0.74,95 \% \mathrm{CI}=(-0.96,-0.52), \quad P$ $<0.001$ ], and increase HDL-C levels [low-dose: SMD = $0.53,95 \% \mathrm{CI}=(0.33,0.73), P<0.001$; high-dose: $\mathrm{SMD}=$ $0.79,95 \% \mathrm{CI}=(0.38,1.21), P<0.001]$. In the subgroup analyses stratified by the duration of the treatment with Danhong injection, both short- and long-duration of treatment could significantly decrease the levels of TG [short-duration: $\mathrm{SMD}=-0.76,95 \% \mathrm{CI}=(-0.99,-0.53)$, $P<0.001$; long-duration: $\mathrm{SMD}=-0.80,95 \% \mathrm{CI}=(-0$. 99, -0.61$), P<0.001$ ], TC [short-duration: $\mathrm{SMD}=-1$. 12, 95\% CI $=(-1.38,-0.86), P<0.001$; long-duration: $\mathrm{SMD}=-1.42,95 \% \mathrm{CI}=(-1.92,-0.92), P<0.001]$ and LDL-C [short-duration: SMD $=-0.71,95 \% \mathrm{CI}=(-0.92$, 0.50), $P<0.001$; long-duration: $\mathrm{SMD}=-0.85,95 \% \mathrm{CI}$ $=(-1.11,-0.58), \quad P<0.001]$, and increase HDL-C levels [short-duration: $\mathrm{SMD}=0.92,95 \% \mathrm{CI}=(0.55,1.29)$, $P<0.001$; long-duration: $\mathrm{SMD}=0.51,95 \% \mathrm{CI}=(0.07,0.95)$, $P=0.02]$.

Associations of Huangqi injection with plasma lipid levels The outcomes of the analysis on all studies showed that Huangqi injection could significantly decrease TC levels $[\mathrm{SMD}=-1.13,95 \% \mathrm{CI}=(-2.09,-0.16), P=0.02]$, and marginally significantly decrease TG levels $[\mathrm{SMD}=-1$. $27,95 \% \mathrm{CI}=(-2.53,0.00), P=0.05]$. Huangqi injection did not have significant effects on the changes in LDL-C and HDL-C levels (Table 2 and Fig. 5).

\section{Heterogeneity analysis}

In the analysis for Danhong injection, there was significant heterogeneity in the total analyses for TG, TC, LDL-C and HDL-C (Table 1). Thirteen studies [13, 22, $24,29,33-35,54-56,58-60], 20$ studies [14, 16, 17, 21, 26-29, 34, 39-43, 53, 54, 56-58, 62], 9 studies [14, 22, $26,28,29,34,40,41,56]$ and 15 studies [17-26, 34, 35, $40,56,57]$ were respectively identified as the main contributors to the heterogeneity for TG, TC, LDL-C and HDL-C by using Galbraith plots (Additional file 2: Figures S1-S4). The heterogeneity was effectively removed or decreased after exclusion of these outlier studies, but the SMD values and 95\% CIs did not change substantially [TG: $\mathrm{SMD}=-0.80,95 \% \mathrm{CI}=(-0.90,-0.70), P_{\mathrm{SMD}}$ $<0.001, P_{\text {Heterogeneity }}=0.06$; TC: $\mathrm{SMD}=-1.17,95 \% \mathrm{CI}$ $=(-1.28, \quad-1.06), \quad P_{\mathrm{SMD}}<0.001, \quad P_{\text {Heterogeneity }}=0.17$; LDL-C: $\mathrm{SMD}=-0.68,95 \% \mathrm{CI}=(-0.76,-0.61), P_{\mathrm{SMD}}$ $<0.001, P_{\text {Heterogeneity }}=0.26$; HDL-C: $\mathrm{SMD}=0.42,95 \%$ $\left.\mathrm{CI}=(0.29,0.54), P_{\mathrm{SMD}}<0.001, P_{\text {Heterogeneity }}=0.17\right]$.

In the analysis for Huangqi injection, there was significant heterogeneity in the total analyses for TG, TC and HDL-C (Table 2). Three studies [48-50], 3 studies [47, 49, 50] and 1 study [51] were respectively identified as the main contributors to the heterogeneity for TG, TC and HDL-C by using Galbraith plots (Additional file 2: Figures S5-S7). The heterogeneity was effectively removed or decreased after exclusion of these outlier studies, but the SMD values and 95\% CIs did not change substantially [TG: $\mathrm{SMD}=-0.62,95 \% \mathrm{CI}=(-1.17,-0.08), \quad P_{\mathrm{SMD}}=0.03$, $P_{\text {Heterogeneity }}=0.051 ; \mathrm{TC}: \mathrm{SMD}=-0.70,95 \% \mathrm{CI}=(-1.00$, - 0.40), $\quad P_{\mathrm{SMD}}<0.001, \quad P_{\text {Heterogeneity }}=0.17$; HDL-C: $\mathrm{SMD}=-0.02, \quad 95 \% \quad \mathrm{CI}=(-0.29, \quad 0.33), \quad P_{\mathrm{SMD}}=0.46$, $\left.P_{\text {Heterogeneity }}=0.90\right]$. 
Table 1 Meta-analysis of Danhong injection with the changes in plasma lipid levels

\begin{tabular}{|c|c|c|c|c|}
\hline Groups or subgroups & Studies (Subjects) & $P_{\text {Heterogeneity }}$ & SMD $(95 \% \mathrm{Cl})$ & $P_{\text {SMD }}$ \\
\hline \multicolumn{5}{|l|}{ Triglycerides } \\
\hline All & $38(4213)$ & $<0.001$ & $-0.76(-0.91,-0.61)$ & $<0.001$ \\
\hline Coronary artery disease & $7(790)$ & $<0.001$ & $-0.52(-0.88,-0.16)$ & $<0.01$ \\
\hline Cerebrovascular disease & $16(2218)$ & $<0.001$ & $-0.94(-1.13,-0.74)$ & $<0.001$ \\
\hline Diabetes & $9(695)$ & 0.001 & $-0.58(-0.87,-0.29)$ & $<0.001$ \\
\hline Hyperlipidemia & $5(444)$ & $<0.001$ & $-0.87(-1.54,-0.20)$ & $<0.05$ \\
\hline Low-dose ( $\leq 20 \mathrm{~mL})$ & $13(1257)$ & $<0.001$ & $-0.90(-1.15,-0.66)$ & $<0.001$ \\
\hline High-dose (> 20 mL) & $25(2956)$ & $<0.001$ & $-0.69(-0.88,-0.50)$ & $<0.001$ \\
\hline Short-duration ( $\leq 2$ weeks) & $20(2472)$ & $<0.001$ & $-0.76(-0.99,-0.53)$ & $<0.001$ \\
\hline Long-duration (> 2 weeks) & $17(1661)$ & $<0.001$ & $-0.80(-0.99,-0.61)$ & $<0.001$ \\
\hline \multicolumn{5}{|l|}{ Total cholesterol } \\
\hline All & $41(4781)$ & $<0.001$ & $-1.29(-1.56,-1.03)$ & $<0.001$ \\
\hline Coronary artery disease & $7(982)$ & $<0.001$ & $-1.12(-1.85,-0.40)$ & $<0.01$ \\
\hline Cerebrovascular disease & $18(2390)$ & $<0.001$ & $-1.53(-1.94,-1.13)$ & $<0.001$ \\
\hline Diabetes & $9(695)$ & $<0.001$ & $-1.28(-1.87,-0.70)$ & $<0.001$ \\
\hline Hyperlipidemia & $6(648)$ & 0.08 & $-1.16(-1.46,-0.86)$ & $<0.001$ \\
\hline Low-dose ( $\leq 20 \mathrm{~mL})$ & $13(1449)$ & $<0.001$ & $-1.13(-1.49,-0.78)$ & $<0.001$ \\
\hline High-dose (> 20 mL) & $28(3332)$ & $<0.001$ & $-1.38(-1.73,-1.02)$ & $<0.001$ \\
\hline Short-duration ( $\leq 2$ weeks) & $21(2544)$ & $<0.001$ & $-1.12(-1.38,-0.86)$ & $<0.001$ \\
\hline Long-duration (> 2 weeks) & $19(2157)$ & $<0.001$ & $-1.42(-1.92,-0.92)$ & $<0.001$ \\
\hline \multicolumn{5}{|l|}{ LDL-C } \\
\hline All & $38(4412)$ & $<0.001$ & $-0.76(-0.93,-0.59)$ & $<0.001$ \\
\hline Coronary artery disease & $8(1012)$ & $<0.001$ & $-0.72(-1.15,-0.30)$ & 0.001 \\
\hline Cerebrovascular disease & $16(2183)$ & $<0.001$ & $-0.94(-1.24,-0.64)$ & $<0.001$ \\
\hline Diabetes & $7(503)$ & 0.02 & $-0.56(-0.86,-0.26)$ & $<0.001$ \\
\hline Hyperlipidemia & $5(444)$ & 0.34 & $-0.54(-0.74,-0.34)$ & $<0.001$ \\
\hline Low-dose ( $\leq 20 \mathrm{~mL})$ & 14 (1579) & $<0.001$ & $-0.79(-1.05,-0.53)$ & $<0.001$ \\
\hline High-dose (> 20 mL) & $24(2833)$ & $<0.001$ & $-0.74(-0.96,-0.52)$ & $<0.001$ \\
\hline Short-duration ( $\leq 2$ weeks) & $18(2115)$ & $<0.001$ & $-0.71(-0.92,-0.50)$ & $<0.001$ \\
\hline Long-duration (> 2 weeks) & $19(2217)$ & $<0.001$ & $-0.85(-1.11,-0.58)$ & $<0.001$ \\
\hline \multicolumn{5}{|l|}{$\mathrm{HDL}-\mathrm{C}$} \\
\hline All & $32(3616)$ & $<0.001$ & $0.70(0.41,0.98)$ & $<0.001$ \\
\hline Coronary artery disease & $5(500)$ & $<0.001$ & $0.75(-0.00,1.50)$ & 0.05 \\
\hline Cerebrovascular disease & $17(2318)$ & $<0.001$ & $0.98(0.55,1.41)$ & $<0.001$ \\
\hline Diabetes & $4(288)$ & 0.10 & $0.32(-0.07,0.70)$ & 0.10 \\
\hline Hyperlipidemia & $5(444)$ & $<0.001$ & $0.19(-0.32,0.69)$ & 0.47 \\
\hline Low-dose ( $\leq 20 \mathrm{~mL})$ & $11(1032)$ & $<0.01$ & $0.53(0.33,0.73)$ & $<0.001$ \\
\hline High-dose (> 20 mL) & $21(2584)$ & $<0.001$ & $0.79(0.38,1.21)$ & $<0.001$ \\
\hline Short-duration ( $\leq 2$ weeks) & 16 (2070) & $<0.001$ & $0.92(0.55,1.29)$ & $<0.001$ \\
\hline Long-duration (> 2 weeks) & 15 (1466) & $<0.001$ & $0.51(0.07,0.95)$ & 0.02 \\
\hline
\end{tabular}

SMD standardized mean difference, 95\% Cl 95\% confidence interval, TG triglycerides, TC total cholesterol, LDL-C low-density lipoprotein cholesterol, HDL-C highdensity lipoprotein cholesterol 


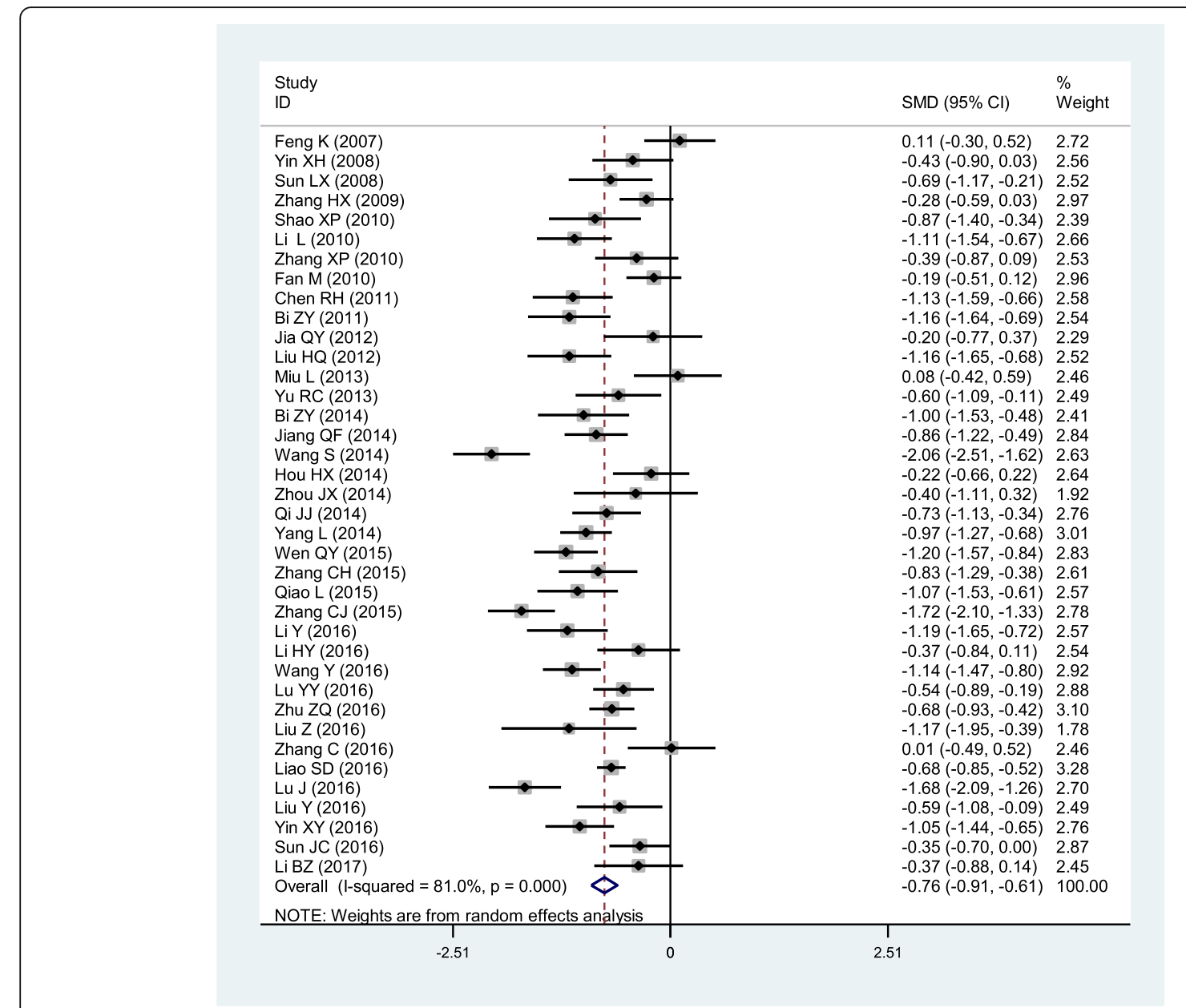

Fig. 1 Forest plot of the meta-analysis between Danhong injection and the change in plasma TG levels

\section{Publication bias test}

Begg's test and funnel plot were used to detect the potential publication bias, and no evidence of significant publication bias was detected in the analyses for Danhong injection (TG: $Z=0.65, P=0.51$; TC: $Z=1.74, P=0.08$; LDL-C: $Z=0.03, \quad P=0.98 ;$ HDL-C: $Z=1.48, \quad P=0.14$ ) (Additional file 2: Figures S8-S11), and for Huangqi injection (TG: $Z=0.38, P=0.71$; TC: $Z=1.50, P=0.13$; LDL-C: $Z=0.00, P=1.00$; HDL-C: $Z=0.00, P=1.00$ ) (Additional file 2: Figures S12-S15).

\section{Discussion}

A substantial number of clinical trials have investigated the lipid-lowering effects of Danhong and Huangqi injections. Associations of the two injections with decreased levels of TG, TC and LDL-C, and/or increased level of HDL-C have been reported in some, but not all studies. The lack of consistency across these studies reflects some limitations such as small sample size and differences in dose and duration of treatment. In the present meta-analysis, the lipid-lowering effects of
Danhong and Huangqi injections were investigated to clarify these discrepancies. To our knowledge, the present study is the first meta-analysis to explore the lipid-lowering effects of Danhong and Huangqi injections based on RCTs.

The results of this meta-analysis suggested that Danhong injection could effectively decrease plasma TG, TC and LDL-C levels, and increase HDL-C level in the total population. In subgroup analyses, we found that Danhong injection could decrease TG, TC, LDL-C, and increase HDL-C levels in CHD and stroke patients. In diabetic and hyperlipidemic patients, Danhong injection could significantly decrease TG, TC and LDL-C levels, but had no significant effect on HDL-C level. The lipidlowering effects of Danhong injection were very robust, which did not vary greatly when the analysis was stratified by dose and duration of treatment. The present meta-analysis also demonstrated that Huangqi injection could decrease plasma levels of TG and TC, but the effects of Huangqi injection on plasma lipid levels need to be further explored with large sample size. 


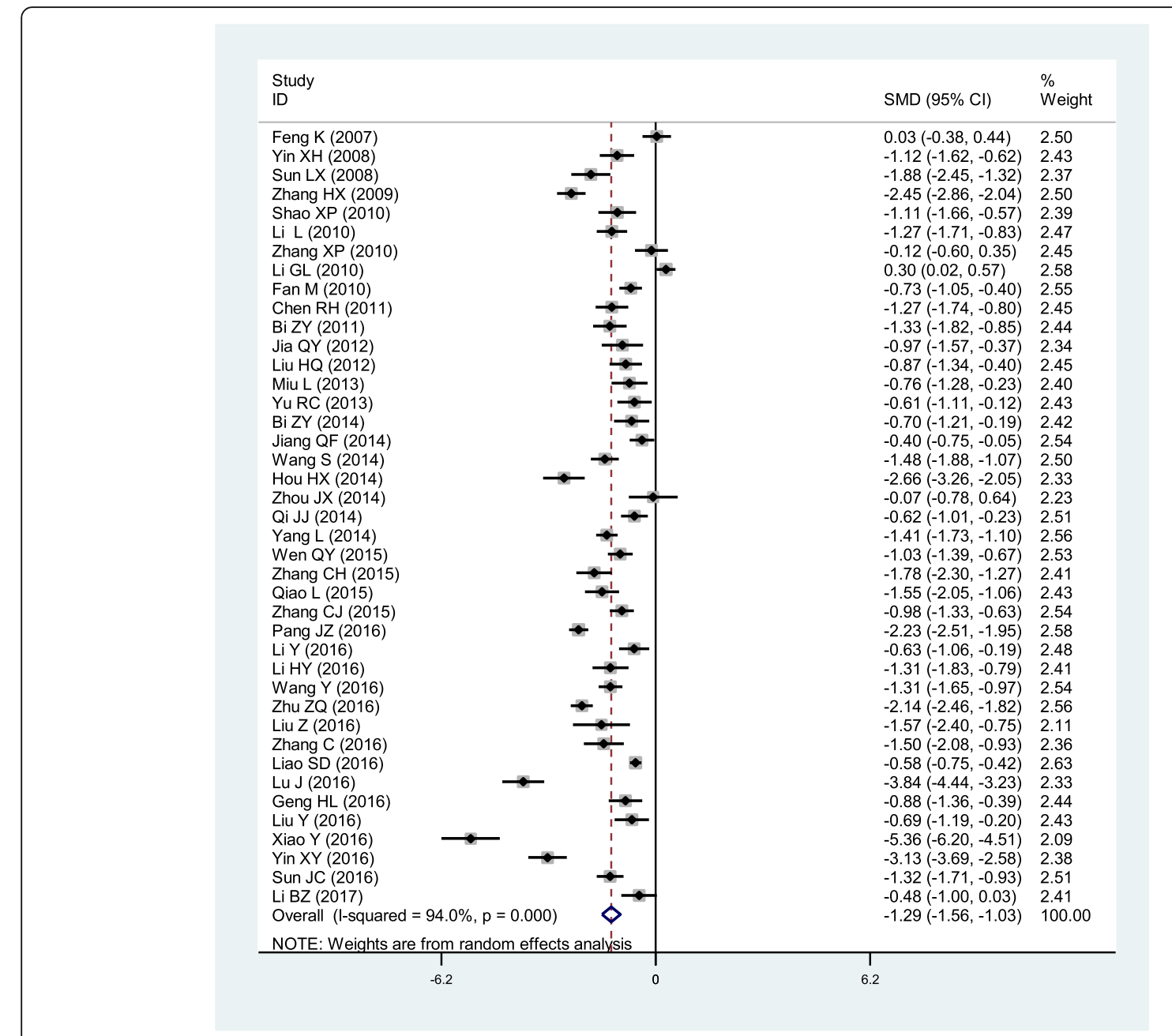

Fig. 2 Forest plot of the meta-analysis between Danhong injection and the change in plasma TC levels

The mechanisms by which Huangqi injection lowers plasma lipid levels have not been clarified yet. However, several studies [64, 65] have investigated the potential mechanisms by which Danhong injection reduced plasma lipid levels. Chen J et al. [64] demonstrated that the lipid-lowering effects of Danhong injection were mediated by up-regulation of the lipolytic genes including carnitine palmitoyl transferase $1(C P T 1)$ and peroxisome proliferator-activated receptor alpha (PPARA), and down-regulation of the lipogenic genes including fatty acid synthase $(F A S)$ and hydroxymethylglutaryl-CoA reductase $(H M G C R)$. The upregulation of adenosine triphosphate-binding cassette transporter A1 (ABCA1), a key factor in reverse cholesterol transport pathway, by Danhong injection was also reported [65].

The duration of the treatment with Danhong and Huangqi injections was varied from 1 week to 6 months in the present meta-analysis. By searching the databases such as PubMed, Google Scholar, Web of Science, Cochrane Library, Wanfang Database, CNKI and VIP, we did not find any studies which had the treatment duration longer than 6 months. By analyzing the original data from the studies included in the present metaanalysis, we found that the treatment duration of Danhong injection had profound effects on blood lipid levels. For example, Danhong injection could decrease TG, TC and LDL-C by $12-17 \%$ after 2 weeks of treatment, $20-25 \%$ after 1 to 2 months of treatment and remained at these levels thereafter. It could increase HDL-C by $14-16 \%$ after 2 weeks of treatment, $21-24 \%$ after 1 to 2 months of treatment, and $37-40 \%$ after 5 to 6 months of treatment. Regarding Huangqi injection, it could decrease TG, TC and LDL-C by $8-13 \%$ after 2 weeks to 1 month of treatment, and $8-18 \%$ after 6 months of treatment. Huangqi injection had no effect on HDL-C levels.

None of the included studies in the present metaanalysis has mentioned the side effects of Danhong injection. However, one study [49] pointed out that Huangqi injection had a side effect of xerostomia in a few patients. 


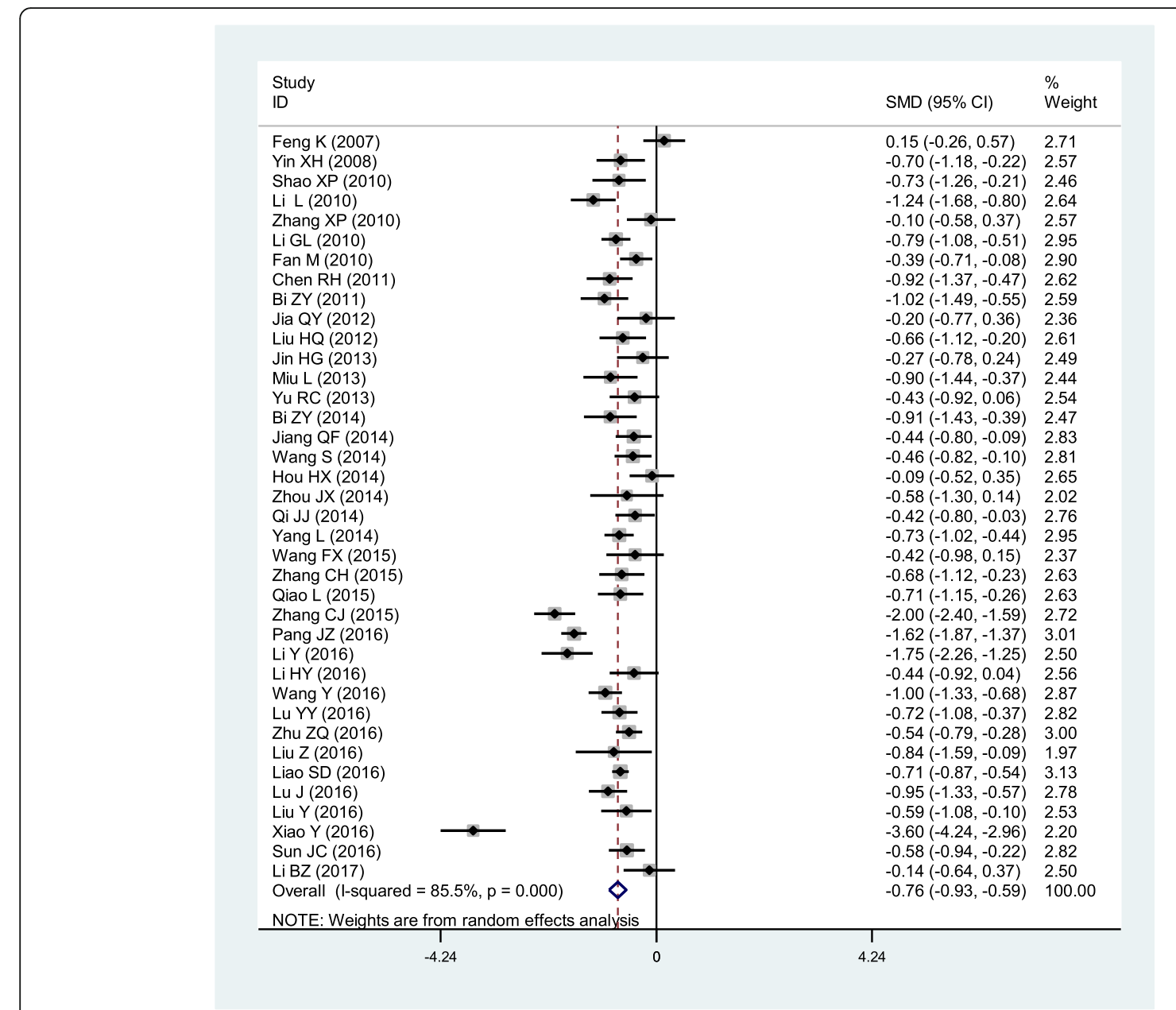

Fig. 3 Forest plot of the meta-analysis between Danhong injection and the change in plasma LDL-C levels

In addition, several studies [66-69] which specifically focused on the side effects of Danhong and Huangqi injections demonstrated that there might be some side effects of Danhong injection such as skin pruritus, flushing, rash, injection site swelling, nausea and vomiting, and of Huangqi injection such as vomiting, rash, fever, dyspnea and bosom frowsty. No toxicology has been reported for Danhong or Huangqi injection.

U.S. Food and Drug Administration (USFDA) promulgated Good Laboratory Practices (GLP) in 1979, and the guidelines of which gradually became the international standards to ensure uniformity, consistency, reliability, reproducibility, quality, and integrity of chemical (including pharmaceuticals) non-clinical safety tests. Based on the GLP guidelines of USFDA and combined with the actual situations in China, China Food and Drug Administration (CFDA) promulgated Chinese GLP in 2003, and last updated in September, 2017 to ensure drug safety and to avoid the occurrence of drug damage to the greatest extent. Chinese GLP has a set of strict guidelines for drugs such as new drug approval process, adverse drug reaction monitoring and drug instruction supervision. The certification steps of new drugs in China include: application, acceptance, data review, onsite inspection, audit, announcement and so on. In the process of clinical use, the government and the relevant functional departments will also follow up on the safety, efficacy, rational drug use and combined drug use. Danhong and Huangqi injections had to fulfill all the demands and guidelines mentioned above before they could be put into clinical use. The Danhong injection (national medicine permission number: Z20026866; national quality standard: $\geq 5 \mathrm{mg}$ total flavonoids per $\mathrm{mL}$ ) in the present meta-analysis was mainly made by Jinan Buchang Pharmaceutical Co., Ltd. P. R. China, Heze Buchang Pharmaceutical Co., Ltd. P. R. China and Xianyang Buchang Pharmaceutical Co., Ltd. P. R. China. The Huangqi injection (national medicine permission number: Z20003189; national quality standard: $\geq 2 \mathrm{mg}$ astragaloside IV per $\mathrm{mL}$ ) in the present meta-analysis was mainly made by Chengdu Di'ao Jiuhong Pharmaceutical Co., Ltd. P. R. China, Jiangsu Jiuxu Pharmaceutical 


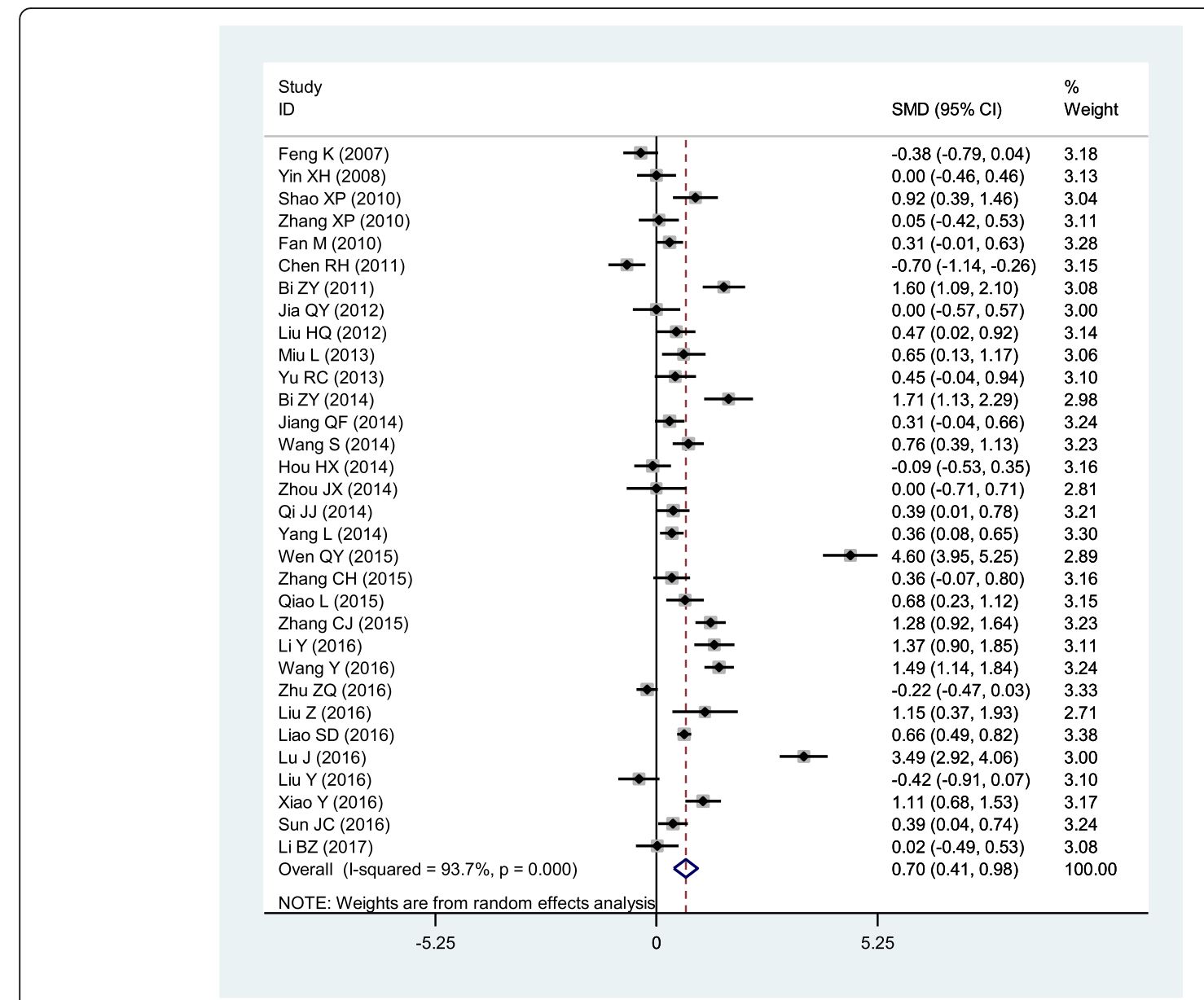

Fig. 4 Forest plot of the meta-analysis between Danhong injection and the change in plasma HDL-C levels

Co., Ltd. P. R. China and Shijiazhuang Shenwei Pharmaceutical Co., Ltd. P. R. China.

With regard to lipid-lowering effects, there was no study that compared Huangqi injection with statins or other drugs. However, two studies [20,70] investigated the lipid-lowering effects of Danhong injection as compared with statins. In a hospital-based study, Bi ZY [20] demonstrated that the lipid-lowering abilities of Danhong injection $(40 \mathrm{~mL} / \mathrm{d})$ were very similar to those of atorvastatin $(10 \mathrm{mg} / \mathrm{d})$, in which TC, LDL-C and TG were significantly reduced, and HDL-C was significantly increased to the same degree with both drugs after 6 months of treatment. Fan HJ et al. [70] compared the lipidlowering abilities of Danhong injection (at doses of $1.0 \mathrm{~mL} /$ $\mathrm{kg}, 2.0 \mathrm{~mL} / \mathrm{kg}, 4.0 \mathrm{~mL} / \mathrm{kg})$ with simvastatin $(2.0 \mathrm{mg} / \mathrm{kg})$ in rats and found that at the dose of $4.0 \mathrm{~mL} / \mathrm{kg}$, Danhong injection had comparable lipid-lowering effects as simvastatin.

Significant heterogeneity was detected in the analysis for Danhong injection (TG, TC, LDL-C and HDL-C) and Huangqi injection (TG and TC). Subgroup analysis stratified by the health status of subjects, dose and duration of treatment was performed to explore the potential sources of the observed heterogeneity, and the results showed that the main sources of heterogeneity were from the health status of subjects, dose and duration of treatment. Galbraith plot was employed to figure out the studies which produced heterogeneity. Outlier studies were identified by using the plots, and heterogeneity was effectively removed or decreased after excluding the outlier studies. No significant changes in SMD values and $95 \%$ CIs were found after excluding the outlier studies. The results from this meta-analysis were based on random effects model. Comparing with fixed effects model, the random effects model is a more conservative method and less likely to produce false-positive results. Funnel plots and Begg's tests showed no publication bias for all of the lipid parameters.

Several limitations should be acknowledged in this meta-analysis. Firstly, all the included studies used an A versus $A+B$ design in which patients were randomized to receive a control treatment (control group) or a control treatment plus an experimental treatment (treatment group). This kind of design is likely to generate 
Table 2 Meta-analysis of Huangqi injection with the changes in plasma lipid levels

\begin{tabular}{|c|c|c|c|c|}
\hline Groups or subgroups & Studies (Subjects) & $P_{\text {Heterogeneity }}$ & SMD $(95 \% \mathrm{Cl})$ & $P_{\mathrm{SMD}}$ \\
\hline \multicolumn{5}{|l|}{ TG } \\
\hline All & $6(440)$ & $<0.001$ & $-1.27(-2.53,0.00)$ & 0.05 \\
\hline Nephrotic syndrome & $4(274)$ & $<0.001$ & $-1.49(-3.56,0.58)$ & 0.16 \\
\hline Low-dose (< $40 \mathrm{~mL})$ & $2(196)$ & $<0.001$ & $-3.49(-7.55,0.57)$ & 0.09 \\
\hline High-dose ( $\geq 40 \mathrm{~mL}$ ) & $4(244)$ & $<0.001$ & $-0.21(-1.14,0.73)$ & 0.67 \\
\hline Short-duration ( $<4$ weeks) & $2(160)$ & $<0.001$ & $-2.28(-8.74,4.19)$ & 0.49 \\
\hline Long-duration ( $\geq 4$ weeks) & $4(280)$ & $<0.01$ & $-0.84(-1.43,-0.26)$ & $<0.01$ \\
\hline \multicolumn{5}{|l|}{$\mathrm{TC}$} \\
\hline All & $7(542)$ & $<0.001$ & $-1.13(-2.09,-0.16)$ & 0.02 \\
\hline Nephrotic syndrome & $5(376)$ & $<0.001$ & $-1.41(-2.88,0.07)$ & 0.06 \\
\hline Low-dose $(<40 \mathrm{~mL})$ & $3(298)$ & $<0.001$ & $-2.13(-3.98,-0.29)$ & 0.02 \\
\hline High-dose ( $\geq 40 \mathrm{~mL}$ ) & $4(244)$ & $<0.001$ & $-0.40(-1.47,0.67)$ & 0.47 \\
\hline Short-duration (< 4 weeks) & $2(160)$ & $<0.001$ & $-1.98(-7.85,3.89)$ & 0.51 \\
\hline Long-duration ( $\geq 4$ weeks) & $5(382)$ & 0.03 & $-0.83(-1.19,-0.47)$ & $<0.001$ \\
\hline \multicolumn{5}{|l|}{ LDL-C } \\
\hline All & $3(220)$ & 0.72 & $-0.15(-0.41,0.12)$ & 0.28 \\
\hline \multicolumn{5}{|l|}{$\mathrm{HDL}-\mathrm{C}$} \\
\hline All & $3(220)$ & $<0.05$ & $0.28(-0.22,0.77)$ & 0.27 \\
\hline
\end{tabular}

SMD standardized mean difference, 95\% Cl 95\% confidence interval, TG triglycerides, TC total cholesterol, $L D L-C$ low-density lipoprotein cholesterol, HDL-C highdensity lipoprotein cholesterol

false positive results [71]. However, it was not possible to find well designed trials to evaluate the lipid-lowering effects of Danhong or Huangqi injection. Secondly, a relatively small number of subjects were included in the analysis for Huangqi injection, which may reduce the statistic power and even cause type I error (false-positive results). Further studies with large sample size are required to investigate the lipid-lowering effects of Huangqi injection. Thirdly, this meta-analysis only included the studies published in Chinese, and all of the subjects included were Chinese as there were no studies being conducted outside of China so far. Due to this limitation, the results of this study may only apply to Chinese populations, but cannot be extended to populations elsewhere.

In summary, the present meta-analysis demonstrated that Danhong injection can reduce plasma levels of TG, TC and LDL-C and increase HDL-C level. Huangqi injection also has significant effects on TG and TC, but not as powerful as Danhong injection.

\section{Methods}

\section{Identification and eligibility of relevant studies}

All articles published before April 2017 on the effects of Danhong and Huangqi injections on plasma lipid levels were identified. The languages of the articles were limited to English and Chinese. A comprehensive search of the literature was carried out by using the databases including PubMed, Google Scholar, Web of Science, Cochrane Library, Wanfang, CNKI and VIP. The keywords used for this search were "Danhong or Huangqi or Danhong injection or Huangqi injection or astragalus injection" concatenated with "triglyceride or total cholesterol or high-density lipoprotein cholesterol or lowdensity lipoprotein cholesterol or TG or TC or HDL-C or LDL-C or hyperlipidemia or dyslipidaemia or hypercholesterolemia or hypertriglyceridemia or coronary heart disease or coronary artery disease or stroke or diabetes". The variables of this meta-analysis were limited to TG, TC, LDL-C and HDL-C. The studies that fulfilled the following criteria were included: (1) studies in which an $A$ versus $A+B$ design was used, i.e. a control treatment (control group) versus a control treatment plus an experimental treatment (treatment group); (2) studies being designed as randomized and double-blind clinical trials; (3) studies reporting the effects of Danhong or Huangqi injection on at least one of the four lipid variables (TG, TC, LDL-C and HDL-C); (4) studies in which mean values and standard deviations (SD) or standard errors (SE) were available; (5) studies in which fasting lipid levels were given; (6) lipid data were available before and after the treatment of Danhong or Huangqi injection; (7) lipid data were available for both control group and treatment group. All references cited in the included articles were reviewed to check the published work which was not indexed by PubMed, Google 
a

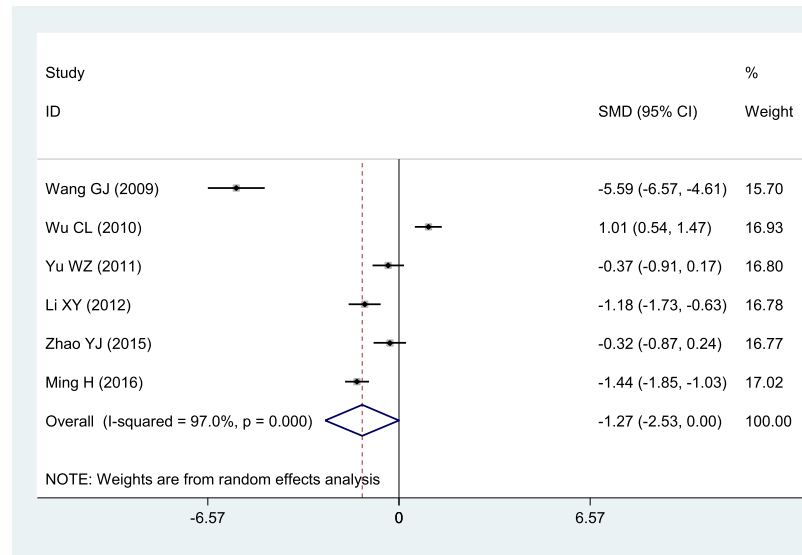

C

Study

ID

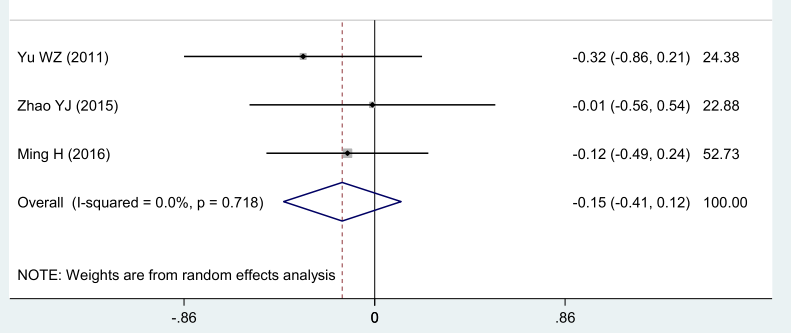

b

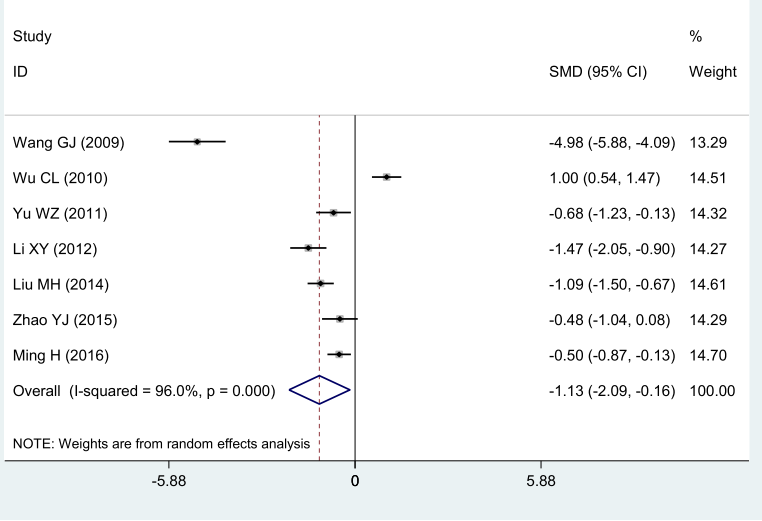

d

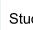

Study

ID

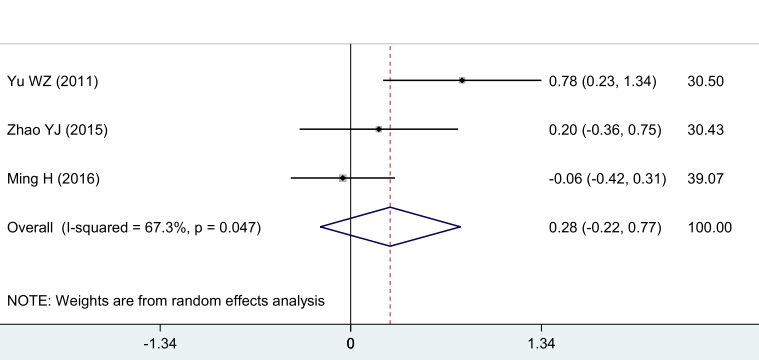

Fig. 5 Forest plots of the meta-analysis between Huangqi injection and the changes in plasma lipid levels. a Forest plot of the meta-analysis between Huangqi injection and the change in plasma TG levels; b Forest plot of the meta-analysis between Huangqi injection and the change in plasma TC levels; c Forest plot of the meta-analysis between Huangqi injection and the change in plasma LDL-C levels; $\mathbf{d}$ Forest plot of the meta-analysis between Huangqi injection and the change in plasma HDL-C levels

Scholar, Web of Science, Cochrane Library, Wanfang, CNKI and VIP databases. Reports with incomplete data, studies based on pedigree data, case reports, review articles, abstracts and animal studies were excluded from the meta-analysis.

\section{Data extraction}

Data were extracted by using a structured data collection form. The irrelevant studies or the studies that did not meet the inclusion criteria were excluded after being reviewed independently by two reviewers. The data were double-checked and compared after extraction. Data uncertainty was discussed and solved by the whole group. For the overlapping articles, only those publications that presented the most detailed information were included. In the present meta-analysis, the data extracted from each of the included studies were as follows: first author, age, gender, ethnicity, health condition, treatment dose, treatment duration, sample size, lipid data before and after the treatment of Danhong or Huangqi injection.

\section{Statistical analysis}

The unit $\mathrm{mmol} / \mathrm{L}$ was used for all lipid variables in the meta-analysis. The standardized mean difference (SMD) for net change and 95\% confidence interval (95\% CI) were used to determine the effects of Danhong or Huangqi injection on plasma levels of TG, TC, LDL-C and HDL-C. The following formula was used to calculate the changes in mean values from pre- to post-treatment: mean change $=$ mean $_{\text {post-treatment }}-$ mean $_{\text {pre-treatment }}$, and the following formula was used to calculate the changes in $\mathrm{SD}$ values: $\mathrm{SD}=$ square root $\left[\left(\mathrm{SD}_{\text {pre-treatment }}\right)^{2}+\left(\mathrm{SD}_{\text {post- }}\right.\right.$ treatment $)^{2}-2 \mathrm{R} \times \mathrm{SD}_{\text {pre-treatment }} \times \mathrm{SD}_{\text {post-treatment }}$, assuming a correlation coefficient $(\mathrm{R})=0.5 \quad$ [72]. The STATA software package (Version 10, Stata Corporation, College Station, TX) was used for all statistical analyses. All data were presented as mean $\pm \mathrm{SD}$ in this meta-analysis. 
Subgroup analyses were conducted according to dose of treatment, duration of treatment and health conditions. Health condition subgroup was defined as CHD, diabetes, stroke and hyperlipidemia.

The random effects model was used in the metaanalysis in that (1) both between-study and within-study heterogeneity is considered in this model; (2) the random effects model provides a more conservative evaluation of the significance of the associations than the fixed effects model [73]. Heterogeneity among studies was tested by Cochran's $X^{2}$-based Q-statistic at a significance level of $P<0.05$. Galbraith plot was used to detect the potential sources of heterogeneity, and the SMD values were recalculated after excluding the outlier studies. Publication bias was assessed by Begg's rank correlation tests and funnel plots, and a significance level of 0.05 was used to indicate the presence of potential publication bias [74].

\section{Additional files}

Additional file 1: Table S1. Characteristics of the studies included in the meta-analysis for Danhong injection; Table S2. Characteristics of the studies included in the meta-analysis for Huangqi injection; Table S3. The lipid level changes from pre- to post-treatment with Danhong injection in control or treatment groups; Table S4. The lipid level changes from pre- to posttreatment with Huangqi injection in control or treatment groups. (DOCX $65 \mathrm{~kb}$ )

Additional file 2: Figures S1-S4. Galbraith plots of the association analysis between Danhong injection and the changes in plasma levels of TG, TC, LDL-C and HDL-C, respectively; Figures S5-S7. Galbraith plots of the association analysis between Huangqi injection and the changes in plasma levels of TG, TC and HDL-C, respectively; Figures S8-S11. Begg's funnel plots of the association analysis between Danhong injection and the changes in plasma levels of TG, TC, LDL-C and $H D L-C$, respectively; Figures S12-S15. Begg's funnel plots of the association analysis between Huangqi injection and the changes in plasma levels of TG, TC, LDL-C and $\mathrm{HDL}-\mathrm{C}$, respectively. (DOCX $54 \mathrm{~kb}$ )

\section{Abbreviations}

95\% Cl: 95\% confidence interval; CHD: Coronary heart disease; HDL-C: Highdensity lipoprotein cholesterol; LDL-C: Low-density lipoprotein cholesterol; SMD: Standardized mean difference; TC: Total cholesterol; TG: Triglycerides

\section{Acknowledgements}

This research was supported by the grants from the Key Project of Education Department of Sichuan Province, P. R. China (17ZA0172) and the grants from the Cooperative Project of Scientific Research between Nanchong city and North Sichuan Medical College, P. R. China (NSMC20170403).

\section{Availability to data and materials}

All data generated or analysed during this study are included in this published article [and its supplementary information files].

\section{Funding}

This research was supported by the grants from the Key Project of Education Department of Sichuan Province, P. R. China (17ZA0172) and the grants from the Cooperative Project of Scientific Research between Nanchong city and North Sichuan Medical College, P. R. China (NSMC20170403).

\section{Authors' contributions}

SYY, YLQ and ZCY conceived of the study, participated in the design, and drafted the manuscript. YLQ, LZ, ZW, LFY and HGJ carried out the study searches and collected the data. SYY performed the statistical analysis. All authors read and approved the final manuscript.
Ethics approval and consent to participate

Not applicable

\section{Competing interests}

The authors declare that they have no competing interests.

\section{Publisher's Note}

Springer Nature remains neutral with regard to jurisdictional claims in published maps and institutional affiliations.

\section{Author details}

${ }^{1}$ Institute of Materia Medica, School of Pharmacy, North Sichuan Medical College, Nanchong 637000, People's Republic of China. ²Department of Cardiology, Affiliated Hospital of North Sichuan Medical College, Nanchong 637000, People's Republic of China. ${ }^{3}$ School of Clinical Medicine, North Sichuan Medical College, Nanchong 637000, People's Republic of China. ${ }^{4}$ School of Preclinical Medicine, North Sichuan Medical College, and Nanchong Industry Technology Institute of BioMedicine, Nanchong 637000, People's Republic of China.

Received: 1 October 2017 Accepted: 26 April 2018

Published online: 10 May 2018

\section{References}

1. Yusuf S, Hawken S, Ounpuu S, Dans T, Avezum A, Lanas F, McQueen M, Budaj A, Pais P, Varigos J, et al. Effect of potentially modifiable risk factors associated with myocardial infarction in 52 countries (the INTERHEART study): case-control study. Lancet. 2004;364:937-52.

2. Abdel-Maksoud M, Sazonov V, Gutkin SW, Hokanson JE. Effects of modifying triglycerides and triglyceride-rich lipoproteins on cardiovascular outcomes. J Cardiovasc Pharmacol. 2008:51:331-51.

3. Padala S, Thompson PD. Statins as a possible cause of inflammatory and necrotizing myopathies. Atherosclerosis. 2012;222:15-21.

4. Young JB, Ghobrial II. Autoimmune statin-induced myopathy: a case report. J Community Hosp Intern Med Perspect. 2015;5:28374.

5. László A, Kalabay L, Nemcsik J. Case report of exercise and statin-fibrate combination therapy-caused myopathy in a patient with metabolic syndrome: contradictions between the two main therapeutic pathways. BMC Res Notes. 2013;6:52.

6. Wu JR, Zhang XM, Zhang B. Danhong injection in the treatment of acute coronary syndrome: a systematic review and meta-analysis. Am J Chin Med. 2015:43:199-214.

7. Liao P, Wang L, Guo L, Zeng R, Huang J, Zhang M. Danhong injection (a traditional (Chinese patent medicine) for acute myocardial infarction: a systematic review and meta-analysis. Evid Based Complement Alternat Med. 2015;2015:646530.

8. Wang K, Zhang D, Wu J, Liu S, Zhang X, Zhang B. A comparative study of Danhong injection and Salvia miltiorrhiza injection in the treatment of cerebral infarction: a systematic review and meta-analysis. Medicine (Baltimore). 2017;96:e7079.

9. Yao JY, Zhi M, Cao WT, Huang Y, Li CJ. Successful treatment with danhong injection for hepatic veno-occlusive disease. HepatoGastroenterology. 2011;58:992-5.

10. Zhu C, Gao Y, Jiang T, Hao C, Gao Z, Sun Y. Meta-analysis of Huangqi injection for the adjunctive therapy of aplastic anemia. Int J Clin Exp Med. 2015;8:10256-64.

11. Han H, Cao A, Wang L, Guo H, Zang Y, Li Z, Zhang X, Peng W. Huangqi decoction ameliorates Streptozotocin-induced rat diabetic nephropathy through antioxidant and regulation of the TGF- $\beta$ /MAPK/PPAR- $\gamma$ signaling. Cell Physiol Biochem. 2017:42:1934-44.

12. Zhang C, Zhu C, Ling Y, Zhou X, Dong C, Luo J, Liu Y. The clinical value of Huangqi injection in the treatment of leucopenia: a meta-analysis of clinical controlled trials. PLoS One. 2013;8:e83123.

13. Jia QY. Effect of Danhong injection on blood lipids in elderly patients. Zhongguo Shi Yong Yi Yao. 2012;7:162-3.

14. Zhang XP, Li XS. Clinical observation on efficacy of Danhong injection in adjuvant therapy of T2DM combined with dyslipidemia. Zhongguo Yao Ye. 2010;19:72-3.

15. Lu YY. Efficacy of Danhong injection combined with atorvastatin in the treatment of unstable angina pectoris of coronary heart disease. Qi Qi Ha Er Yi Xue Yuan Xue Bao. 2016;37:2664-5. 
16. Yin $X Y$. Danhong injection in the treatment of early diabetic nephropathy for 56 cases. Zhongguo Zhong Yi Yao Xian Dai Yuan Cheng Jiao Yu. 2016:58-9.

17. Liao SD, Wang CY, Zhang T. Application of Danhong injection in elderly patients with angina pectoris complicated with cerebral infarction. Shan X Zhong Yi Xue Yuan Xue Bao. 2016;39:28-30.

18. Bi ZY. Study on improving effects of Danhong injection on clinical symptoms, blood lipid and electrocardiogram of patients with angina pectoris. Shi Yong Yu Fang Yi Xue. 2011;18:2347-8.

19. Liu Y. Clinical observation of Danhong injection in the treatment of acute exacerbation of chronic pulmonary heart disease. Shi Zhen Guo Yi Guo Yao. 2016;25:1183-6.

20. Bi ZY. Study on the lipid-lowering effect of atorvastatin combined with Danhong injection in coronary heart disease patients. Si Chuan Yi Xue. 2014;35:131-2

21. Zhu ZQ. Danhong injection combined with fluvastatin in the treatment of coronary heart disease complicated with hyperlipidemia: a clinical observation of 121 cases. Zhongguo Shi Yong Yi Yao. 2016:11:167-8.

22. Zhang CJ, Wang HY. Effect of Danhong injection combined with clopidogrel in treating patients with unstable angina pectoris. Zhongguo Shi Yan Xue Fang Qi Xue Za Zhi. 2015;21:171-4.

23. Chen $\mathrm{YH}$, Song QF, Feng JC, Pan HC, Xiao SH, Wei M. Effect of Danhong injection on endothelial function in hyperlipidemic patients. Ren Min Jun Yi. 2011;54:590-1.

24. Wang Y. Effects of Danhong injection on clinical symptoms, blood lipids and electrocardiogram in patients with coronary heart disease. Yi Liao Zhuang Bei. 2016;29:134-5.

25. Yin XH, Liu CW, Gao H, Li J, Liu T, Chen J. Effect of Danhong injection on cerebral hemodynamics, blood rheology and blood lipid levels in patients with ischemic cerebrovascular disease. Shan Dong Zhong Yi Za Zhi. 2008:27:515-8.

26. Li Y, Tian JB. Clinical observation of shenmai injection combined with Danhong injection in the treatment of acute ischemic stroke. Zhongguo Zhong Yi Yao Ke Ji. 2016;23:445-7.

27. Jiang QF. Efficacy analysis of Danhong injection combined with fluvastatin in the treatment of coronary heart disease complicated with hyperlipidemia. Yi Xue Li Lun Yu Shi Jian. 2014:27:1717-8.

28. Li BZ. Effects of Danhong injection combined with ozagrel on four coagulation indexes and blood lipids in patients with acute cerebral infarction. Xian Dai. Zhong Xi Yi Jie He Za Zhi. 2017;26:49-51.

29. Fan M, Liu FY, Yang Y, Duan SB, Ye Y, Wang GX. Effect of Danhong injection combined with alprostadil injection on early diabetic nephropathy. Zhong Nan Yao Xue. 2010;8:235-7.

30. Liu Z, Sun LP. Observation on clinical effects of Danhong injection combined with alprostadil injection in treatment of type 2 diabetic nephropathy. Zhongguo Yi Yuan Yong Yao Ping Jia Yu Fen Xi. 2016;16:1344-6.

31. Shao XP, Kong L, Wang TG. Effect of Danhong injection on blood lipids and hemorheology in patients with ischemic stroke. Dang Dai Yi Xue. 2010;16:82-3.

32. Liu HQ, Pang XH, An DH. Clinical observation of Danhong injection in the treatment of 35 cases of unstable angina pectoris complicated with hyperlipidemia. Ya Tai Chuan Tong Yi Yao. 2012;8:103-4.

33. Wang S, Zeng R. Clinical observation of effect of Danhong injection combined with atorvasatin in treatment of hyperlipoidemia. Liao Ning Zhong Yi Yao Da Xue Xue Bao. 2014:187-8.

34. Hou HX. Curative observation of using Danhong injection combined isosorbide mononitrate sustained release tablets in the treatment of coronary heart disease. Si Chuan Zhong Yi. 2014;32:162-4.

35. Wen QY. Effect of Danhong injection on blood lipid levels in patients with cerebral infarction. Chang Jiang Da Xue Xue Bao (Zi Ke Ban). 2015;12:7-9.

36. Li HY, Zhang LM, Wang YH. Improvement of proteinuria by Danhong injection in patients with type 2 diabetic nephropathy. Zhongguo Chu Fang Yao. 2016:14:75-6

37. Qiao L, Ye R, Xie CR. Effects of Danhong injection combined with high-dose atorvastatin on blood lipids, hs-CRP and TNF-ain patients with acute coronary syndromes undergoing percutaneous coronary intervention. Zhong Yi Yao Dao Bao. 2015;21:49-50.

38. Yang L, Xie JD. Clinical observation of Danhong injection combined with simvastatin in the treatment of 98 cases of coronary heart disease complicated with hyperlipidemia. Zhongguo Xian Dai Yao Wu Ying Yong. 2014;8:152-3.

39. Zhou JX. Clinical observation of Danhong injection in the treatment of early diabetic nephropathy. Si Chuan Zhong Yi. 2014;32:161-2.
40. Xiao Y, Li YQ, Li HJ, Wu YJ. The clinical effect of CDSP treatment for coronary heart disease. Kun Ming Yi Ke Da Xue Xue Bao. 2016;37:84-6.

41. Pang ZJ, Zhang SZ. Effective observation on treating chronic heart failure of coronary heart disease with atorvastatin plus the Danhong injection. Zhong Yi Lin Chuang Yan Jiu. 2016;8:114-6.

42. Yu RC, Li P, Mao BH, Zhang Y. Clinical observation of Danhong injection in lipid-lowering effect. Chang Chun Zhong Yi Yao Da Xue Xue Bao. 2013;29:656-7.

43. Sun LX, Zhao M, Wang LF, Liu WL, Tian CL. Clinical observation of Danhong injection in the treatment of diabetic nephropathy complicated with hyperlipidemia. He Bei Zhong Yi. 2008;30:70.

44. Wang FX, Liu XD, Wang HB. Investigation of the clinical effect of Danhong injection on diabeticnephropathy. Tang Niao Bing Xin Shi Jie. 2015;3:5.

45. Jin HG, Zhao M, Qi F. Clinical observation of Danhong injection on carotid atherosclerotic plaque in patients with coronary heart disease. Zhongguo Shi Yong Yi Yao. 2013;8:169-70.

46. Zhao YJ, Jing ZX. Effect of Huangqi injection on renal injury due to primary hypertension. Zhongguo Xun Zheng Xin Xue Guan Yi Xue Za Zhi. 2015;7:248-50.

47. Li XY, Zheng JJ, Zheng XY, Meng Q. Clinical observation on the treatment for diabetic nephropathy by astragalus injection. Zhongguo Shi Yong Yi Yao. 2012;7:3-4.

48. Ming $H$, Xie $H$, Zhang $H$, Ai K. Efficacy of astragalus injection on patients with hemiplegia after ischemic stroke. Zhongguo Kang Fu Li Lun Yu Shi Jian. 2016:22:684-7.

49. Wu CL, Zhang XL. Clinical observation of astragalus injection in the treatment of diabetic nephropathy. Lin Chuang Yi Yao Shi Jian. 2010;20:601-2.

50. Wang GJ, Yang XQ, Zhang JF. Clinical observation of astragalus injection in the treatment of early-stage diabetic nephropathy. Wei Xun Huan Xue Za Zhi. 2009:19:44-5.

51. Yu WZ, Gao GS, He XS. Effect of astragale injection on serum lipids in nephrotic syndrome. Shi Yong Yao Wu Yu Lin Chuan. 2011;14:194-5.

52. Liu MH. Clinical observation of astragalus injection in the treatment of nephrotic syndrome in children. Zhongguo Yi Yao Zhi Nan. 2014;12:181-2.

53. Li GL, Liu ZY, Cui WN, Wang YR. Clinical observation on Danhong injection with benazepril for elderly hypertensive patients with early kidney demage. Zhong Xi Yi Jie He Xin Nao Xue Guan Bing Za Zhi. 2010;8:7-8.

54. Zhang HX. Effects of Danhong injection on blood lipids and hemorheology in patients with unstable angina pectoris. He Bei Zhong Yi. 2009;31:602-3.

55. Zhang C, Lu DF, Wang DY. Clinical observation of Danhong injection combined with compound alpha keto acid in treatment of early diabetic nephropathy. Huai Hai Yi Yao. 2016:34:335-6.

56. Feng K, Ji XB, Qiu WW, Jiang MY, Shen W, Jiang W, Huang H, Gao YX. The effect of Danhong injection on cardiovascular event in earlier period and inflammatory reaction of the patients of ACS with $\mathrm{PCl}$. Zhongguo Wei Xun Huan. 2007;11:390-2.

57. Qi JJ, Ji YL, Liu SF, Zeng X, Zhao GL, Dai JY, Fu SY, Shi GY, Wang LL. Danhong injection combined with simvastatin in the treatment of 54 cases of coronary heart disease complicated with hyperlipidemia. Zhongguo Yao Ye. 2014;23:103-5.

58. Lu J, Li DP, Zhang CR, Hao D. Efficacy analysis of Danhong injection in the treatment of coronary heart disease. Xin Xue Guan Bing Fang Zhi Zhi Shi. 2016;7:51-2.

59. Sun JC. Clinical observation of fluvastatin sodium combined with Danhong injection in the treatment of hyperlipidemia. Ya Tai Chuan Tong Yi Yao. 2016:12:117-8.

60. Miu L, Hu YH. Observation of the curative effect on atorvastatin joint Danhong injection in the treatment of unstable angina pectoris. Zhongguo Yi Yao Dao Bao. 2013;10:78-80.

61. Li L. Effect of Danhong injection on blood lipids, blood coagulation and urine microalbumin in patients with diabetic nephropathy. Zhongguo Min Kang Yi Xue. 2010;22:2079-80.

62. Zhang $\mathrm{CH}$. Clinical effect of simvastatin combined with danhong injection on coronary heart disease complicated with hyperlipidaemia. Shi Yong Xin Nao Fei Xue Guan Bing Za Zhi. 2015;23:127-9.

63. Geng HL. Clinical observation of Danhong injection in the treatment of 72 cases of angina pectoris. World Latest Med Information (Electronic Version). 2016;16:194

64. Chen J, Deng J, Zhang Y, Yang J, He Y, Fu W, Xing P, Wan H. Lipid-lowering effects of Danhong injection on hyperlipidemia rats. J Ethnopharmacol. 2014:154:437-42.

65. Chen Y, Liu M, Zhao T, Zhao B, Jia L, Zhu Y, Zhang B, Gao X, Li G, Li X, et al. Danhong injection inhibits the development of atherosclerosis in both Apoe $^{-}$I $^{-}$and Ldlr ${ }^{-}$- mice. J CardiovasC Pharmacol. 2014;63:441-52. 
66. Fu XR. Analysis of 46 adverse drug reaction cases induced by Huangqi injection. Ya Tai Chuan Tong Yi Yao. 2015;11:141-2.

67. Wang $L H$, Zhang ST. Analysis of adverse drug reactions of Huangqi injection in clinical practice. Nei Meng Gu Zhong Yi Yao. 2014;33:61-2.

68. Fang XJ, Fei XX. Cause analysis and preventive measures of the adverse drug reactions of Danhong injection. Xin Zhong Yi. 2015;12:191-3.

69. Luo J, Wang Q, Guo QS. Analysis of 39 adverse drug reaction/event cases induced by Danhong injection. Zhongguo Yi Yao Ke Xue. 2015;5:42-4.

70. Fan $\mathrm{H}$, Li M, Yu L, Jin W, Yang J, Zhang Y, Wan H. Effects of Danhong injection on platelet aggregation in hyperlipidemia rats. J Ethnopharmacol. 2018;212:67-73

71. The European Agency for the Evaluation of Medicinal Products, Human Medicines Evaluation Unit. Note for guidance on clinical investigation of medicinal products for the treatment of cardiac failure. In: Technical report; 1999.

72. Liu C, Sun J, Lu Y, Bo Y. Effects of anthocyanin on serum lipids in dyslipidemia patients: a systematic review and meta-analysis. PLoS One. 2016;11:e0162089.

73. Fleiss $\mathrm{J}$. The statistical basis of meta-analysis. Stat Methods Med Res. 1993;2:121-45.

74. Begg CB, Mazumdar M. Operating characteristics of a rank correlation test for publication bias. Biometrics. 1994;50:1088-101.

Ready to submit your research? Choose BMC and benefit from:

- fast, convenient online submission

- thorough peer review by experienced researchers in your field

- rapid publication on acceptance

- support for research data, including large and complex data types

- gold Open Access which fosters wider collaboration and increased citations

- maximum visibility for your research: over $100 \mathrm{M}$ website views per year

At BMC, research is always in progress.

Learn more biomedcentral.com/submissions 